\title{
Some Instances of Homomesy Among Ideals of Posets
}

\author{
Shahrzad Haddadan \\ https://sites.google.com/view/shahrzadhaddadan
}

Submitted: Apr 4, 2016; Accepted: Feb 16, 2021; Published: Mar 26, 2021

(C) The author. Released under the CC BY-ND license (International 4.0).

\begin{abstract}
Given a permutation $\tau$ defined on a set of combinatorial objects $S$, together with some statistic $f: S \rightarrow \mathbb{R}$, we say that the triple $\langle S, \tau, f\rangle$ exhibits homomesy if $f$ has the same average along all orbits of $\tau$ in $S$. This phenomenon was observed by Panyushev (2007) and later studied, named and extended by Propp and Roby (2013). Propp and Roby studied homomesy in the set of order ideals in the product of two chains, with two well known permutations, rowmotion and promotion, the statistic being the size of the order ideal. In this paper we extend their results to generalized rowmotion and promotion, together with a wider class of statistics in the product of two chains. Moreover, we derive similar results in other simply described posets. We believe that the framework we set up here can be fruitful in demonstrating homomesy results in order ideals of broader classes of posets.
\end{abstract}

Mathematics Subject Classifications: 05E18, 06A11

\section{Introduction}

Consider a poset $\mathcal{P}$, and let $J(\mathcal{P})$ be the set containing all of the order ideals in $\mathcal{P}$. Various bijections can be defined mapping $J(\mathcal{P})$ to itself. Among these mappings, the rowmotion (Definition 1) operation has been studied widely by combinatorialists $[6,17$, 5], and under various names (Brouwer-Schrijver map [3], the Fon-der-Flaass map [15], the reverse map [10], and Panyushev complementation [1]). Another mapping called promotion (Definition 2.6) has been defined in analogy to rowmotion. Propp and Roby [14] were interested in studying the orbits these bijections introduce on $J(\mathcal{P})$ and the statistics that are preserved along these orbits. They introduced a concept they called homomesy (Definition 2.8) which is defined on a triple consisting of a set $S$, a bijection on $S$, and a statistic on $S$, that is, a function from $S$ to $\mathbb{R}$. They studied homomesy of promotion and rowmotion on $J(\mathcal{P})$, where $\mathcal{P}$ is a product of two chains.

After Propp and Roby's paper, homomesy has received a lot of attention, and a number of mathematicians have been intrigued by it $[18,5,8,9,2,7,16,12,13]$. While seeming ubiquitous, homomesy is often surprisingly non-trivial to prove. 
Striker and Williams [17] generalized the definition of rowmotion and promotion (see Definitions 2.5 and 2.7) and proved interesting qualities of their orbit structures on the so called rc-posets.

In Section 2, we will provide the formal definition of all the above concepts, along with the statements of our main results. Our proofs are based on an orbit preserving bijection between the order ideals of a poset and sequences of natural numbers. In Section 3, we will present this bijection, and finally the proofs will appear in Section 4.

\section{Our Contribution}

Following the work of Propp and Roby [14] and Striker and Williams [17], we study rowmotion, promotion and their generalizations on $J(\mathcal{P})$ where $\mathcal{P}$ is one of the following three posets: the product of two chains, the upper triangle of this product, or the right side triangle. We introduce a new bijection on the above sets called comotion (Definition 2.9). The main ingredient of the function comotion is a map on order ideals known as the toggle map (Definition 2.1). In Theorems 2.3, 2.4, and 2.5 we characterize a wide class of functions from $J(\mathcal{P})$ to the real numbers which exhibit homomesy along the orbits of comotion.

Our results generalize Propp and Roby's results [14] in three ways: (1) Comotion captures rowmotion and promotion and our results reproduce results of Propp and Roby [14], and generalize them. (2) Our results are not restricted to the order ideals in the product of two chains but also the upper triangle of the product, and the right side triangle. (3) We introduce a set of functions such that any linear combination of them will constitute a homomesic static in our setting, and we observe that the functions studied by Propp and Roby can be constituted using linear combinations of these functions.

At the end of this introduction, we would like to mention that the operation "winching" (Definition 3.1 ) and the Theorems 3.1, 3.2, 3.3 are presented here to aid in understanding comotion on order ideals. In addition, since they are neat examples of homomesy, they may be of independent interest.

\section{Previous Work, Definitions and Statements of Results}

Definition 1. Given a poset $\mathcal{P}$ on the elements of set $\mathcal{S}$, and an order ideal $I \in J(\mathcal{P})$, the rowmotion of $I$, denoted $\Phi(I)$, is defined to be the order ideal generated by the minimal elements in $\mathcal{S}-I$.

Another interesting operation mapping $J(\mathcal{P})$ to itself, first defined by Cameron and Fon-der-Flaass [4], is the toggle map. Toggling is defined as follows:

Definition $2.1([4])$. Given poset $\mathcal{P}$ on the elements of set $\mathcal{S}$, an order ideal $I \in J(\mathcal{P})$, 
and an element $x \in \mathcal{S}$, the toggle map $\sigma_{x}: J(\mathcal{P}) \rightarrow J(\mathcal{P})$ is defined by:

$$
\sigma_{x}(I)= \begin{cases}I \cup\{x\}, & \text { if } x \notin I \text { and } I \cup\{x\} \in J(\mathcal{P}) . \\ I-\{x\}, & \text { if } x \in I \text { and } I-\{x\} \in J(\mathcal{P}) \\ I, & \text { otherwise. }\end{cases}
$$

Cameron and Fon-der-Flaass [4] also noted that each toggle is an involution and gave a criterion for when toggles commute.

Proposition 2.1 ([4]). For all $x \in \mathcal{S}$ and $I \in J(\mathcal{P}), \sigma_{x}^{2}(I)=I$. If $x, y \in \mathcal{S}$, and $x$ does not cover $y$, and $y$ does not cover $x$, we have $\sigma_{x} \circ \sigma_{y}(I)=\sigma_{y} \circ \sigma_{x}(I)$.

A linear extension $\left(x_{1}, \ldots, x_{n}\right)$ of $\mathcal{P}$ is an indexing of the elements of $\mathcal{P}$ satisfying $x_{i}<_{\mathcal{P}} x_{j}$ implies $i<j$. Cameron and Fon-der-Flaass [4] observed that the rowmotion operation coincides with a series of compositions of the toggle map as follows:

Proposition $2.2([4])$. Given an arbitrary $I \in J(\mathcal{P})$ and linear extension $\left(x_{1}, \ldots, x_{n}\right)$ of $\mathcal{P}$, we have $\Phi(I)=\sigma_{x_{1}} \circ \sigma_{x_{2}} \circ \sigma_{x_{3}} \circ \cdots \circ \sigma_{x_{n}}(I)$.

Let $Q_{a, b}=[a] \times[b]$, where $[n]=\{1,2, \ldots, n\}$. We present each element of $\mathcal{Q}_{a, b}$ by a pair $(i, j), i \in[a], j \in[b]$ and we say $\left(i_{1}, j_{1}\right) \leqslant\left(i_{2}, j_{2}\right)$ if and only if $i_{1} \leqslant i_{2}$ and $j_{1} \leqslant j_{2}$. In this paper, we are interested in bijections on $J\left(\mathcal{Q}_{a, b}\right)$, as well as $J\left(\mathcal{U}_{a}\right)$ and $J\left(\mathcal{L}_{a}\right)$ where $\mathcal{U}_{a}$ and $\mathcal{L}_{a}$ are subsets of $\mathcal{Q}_{a, a}$ and defined as:

Definition 2.2. The upper triangle lattice $\mathcal{U}_{a}$ and the right side triangle lattice $\mathcal{L}_{a}$ are two sublattices of $\mathcal{Q}_{a, a}$ defined respectively as, $\mathcal{U}_{a}=\{(i, j) \mid i, j \in[a], i \geqslant a+1-j\}$ and $\mathcal{L}_{a}=\{(i, j) \mid i, j \in[a], i \geqslant j\}$.

See Figure 1 for examples of upper triangle and right side triangle lattices.

We use the following notation throughout: Let $\mathcal{P}$ be one of $\mathcal{Q}_{a, b}, \mathcal{U}_{a}$ or $\mathcal{L}_{a}$. By saying $(i, j) \in \mathcal{P}$ we are referring to the element in $[a] \times[b]$ with coordinates $i$ and $j$. By saying $x=\left(i_{1}, j_{1}\right) \leqslant_{\mathcal{P}} y=\left(i_{2}, j_{2}\right)$ we mean $x$ is less than or equal to $y$ in $\mathcal{P}$, and we may drop the subscript if no confusion would be introduced.

We call $\mathcal{Q}_{a, b}$ the square lattice or the product of two chains, $\mathcal{U}_{a}$ the upper lattice and $\mathcal{L}_{a}$ the left lattice. Among combinatorists $\mathcal{U}_{a}$ is also known as the root poset of type $A_{a}$, and $\mathcal{L}_{a}$ as the minuscule poset of type $B_{a}$ or $D_{a+1}$ (See [15]).

We employ the following terminology:

Definition 2.3. Let $\mathcal{P}$ be one of $\mathcal{Q}_{a, b}, \mathcal{U}_{a}$ or $\mathcal{L}_{a}$. For any arbitrary $I \in J(\mathcal{P})$,

- We call the set of all points $(i, j) \in \mathcal{P}$ with constant $i+j$ a rank; $R_{c}(I)=\{(i, j) \in$ $I \mid i+j=c\}$.

- We call the set of all points $(i, j) \in \mathcal{P}$ with constant $i-j$ a file; $F_{c}(I)=\{(i, j) \in$ $I \mid i-j=c\}$. 


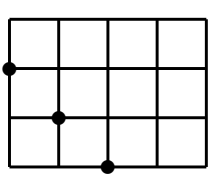

(1)

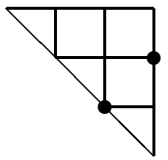

(2)

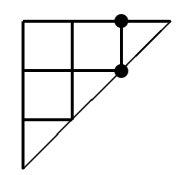

(3)

Figure 1: Examples for Definition 2.2 and 2.3: (1) $\mathcal{Q}_{5,4}$ and $R_{4}\left(\mathcal{Q}_{5,4}\right),(2) \mathcal{U}_{4}$ and $F_{-1}\left(\mathcal{U}_{4}\right)$, (3) $\mathcal{L}_{4}$ and $C_{3}\left(\mathcal{L}_{4}\right)$.

- We call the sets of all points $(i, j) \in \mathcal{P}$ with constant $i$ a column; $C_{c}(I)=\{(i, j) \in$ $I \mid i=c\}$.

When $\mathcal{P}$ is clear from the context, we write $R_{c}=R_{c}(\mathcal{P}), F_{c}=F_{c}(\mathcal{P})$ and $C_{c}=C_{c}(\mathcal{P})$.

See Figure 1 for examples of rank, file and column in different lattices.

We can now define toggling for the above sets.

Definition 2.4. Consider $I \in J(\mathcal{P})$. Take an arbitrary $c$, and let $S$ be one of $R_{c}$ or $F_{c}$. Picking an arbitrary indexing of the elements of $S, x_{1} \ldots x_{m}$ we define $\sigma_{S}(I)=$ $\sigma_{x_{1}} \circ \sigma_{x_{2}} \circ \cdots \circ \sigma_{x_{m}}(I)$. For $S=C_{c}$, we assume $x_{1}<x_{2}<\cdots<x_{m}$ and define $\sigma_{S}(I)=$ $\sigma_{x_{1}} \circ \sigma_{x_{2}} \ldots \sigma_{x_{m}}(I)$.

Note that in the above definition, no two elements $x_{i}, x_{j}$ of $S$ constitute a covering pair, thus by Proposition $2.1 \sigma_{S}$ is well defined.

Striker and Williams studied the class of so-called rc-posets, whose elements are partitioned into ranks and files ${ }^{1}$. Here, we are interested in $\mathcal{Q}_{a, b}, \mathcal{U}_{a}$ or $\mathcal{L}_{a}$ which are all rc-posets. The following definitions are from [17], restricted to the product posets of interest to us. Again, by saying $\mathcal{P}$ we mean one of $\mathcal{Q}_{a, b}, \mathcal{U}_{a}$ or $\mathcal{L}_{a}$ :

Definition $2.5([17])$. Consider a poset $\mathcal{P}$. Let $\nu$ be a permutation of $\{2, \ldots, a+b\}$. We define $\Phi_{\nu}$ to be $\sigma_{R_{\nu(a+b-1)}} \circ \sigma_{R_{\nu(a+b-2)}} \circ \cdots \circ \sigma_{R_{\nu(1)}}$.

Note that by Proposition 2.2, we have $\Phi_{\nu}=\Phi$ for $\nu=(a+b, a+b-1, \ldots, 2)$. Now let $\nu$ be an arbitrary permutation of $\{2, \ldots, a+b\}$. Then, $\Phi_{\nu}$ is a permutation on $J(\mathcal{P})$ and partitions it into orbits. Striker and Williams showed that the orbit structure ${ }^{2}$ of $\Phi_{\nu}$ does not depend on the choice of $\nu[17]$.

We now present another bijection on $J(P)$ which has been studied in [17, 14]:

Definition 2.6. The mapping promotion is a permutation $\partial: J(\mathcal{P}) \rightarrow J(\mathcal{P})$, defined on $I \in J(\mathcal{P})$ as: $\partial(I)=\sigma_{F_{a-1}} \circ \sigma_{F_{a-2}} \circ \cdots \circ \sigma_{F_{0}} \circ \cdots \circ \sigma_{F_{1-b}}(I)$.

\footnotetext{
${ }^{1}$ Striker and Williams use the terminology "row" for what we call "rank" and "column" for what we call "file".

${ }^{2}$ The orbit structure of a bijection $f$ on a set $S$ is the multiset of the sizes of the orbits that bijection $f$ constructs on the set $S$.
} 
As with rowmotion, Striker and Williams [17] define a generalized version of promotion.

Definition $2.7([17])$. Let $\nu$ be a permutation of $\{-b+1, \ldots, a-1\}$. For $I \in J(\mathcal{P})$, we define $\partial_{\nu}(I)$ to be $\sigma_{F_{\nu(a+b-1)}} \circ \sigma_{F_{\nu(a+b-2)}} \circ \cdots \circ \sigma_{F_{\nu(1)}}(I)$.

Note that by Definition 2.6, for $\nu=(-b+1, \ldots, a-1)$ we have $\partial_{\nu}=\partial$.

Like with rowmotion, for any permutation $\nu$ on files of any rc-poset $\mathcal{P}, \partial_{\nu}$ will partition $J(\mathcal{P})$ into orbits. Again, Striker and Williams [17] showed that regardless of which $\nu$ we choose, $J(\mathcal{P})$ will be partitioned into the same orbit structure by $\partial_{\nu}$. Moreover, the orbit structures of $\partial_{\nu}$ and $\Phi_{\omega}$ are the same for any two permutations $\nu$ and $\omega$ :

Theorem 2.1 ([17]). Consider any rc-poset $\mathcal{P}$, for any permutation $\nu$ on $\{2, \ldots, a+b\}$ and $\omega$ on $\{-b+1 \ldots a-1\}$, there is an equivariant bijection between $J(\mathcal{P})$ under $\Phi_{\nu}$ and $J(\mathcal{P})$ under $\partial_{\omega}$.

Note that the above theorem holds in particular for $\mathcal{Q}_{a, b}$ which is a rc-poset.

In 2013, Propp and Roby introduced a phenomenon called homomesy [14]. Propp and Roby discussed some instances of homomesy by studying the actions of promotion and rowmotion on the set $J\left(\mathcal{Q}_{a, b}\right)$. After Propp and Roby's paper, homomesy has attracted the attention of many combinatorialists $[5,8,9,2,7]$, and it is defined as follows:

Definition 2.8 ([14]). Consider a set $S$ of combinatorial objects. Let $\tau: S \rightarrow S$ be a permutation that partitions $S$ into orbits, and $f: S \rightarrow \mathbb{R}$ a statistic of the elements of $S$. We call the triple $\langle S, \tau, f\rangle$ homomesic (or we say it exhibits homomesy) if and only if there is a constant $c$ such that for any $\tau$-orbit $\mathcal{O} \subset \mathcal{S}$ we have

$$
\frac{1}{|\mathcal{O}|} \sum_{x \in \mathcal{O}} f(x)=c
$$

Equivalently, we can say $f$ is homomesic, $c$-mesic or it exhibits homomesy in $\tau$-orbits of $S$.

The following is easily shown from the definition of homomesy.

Proposition 2.3. Suppose we have a set $S$, a permutation $\tau: S \rightarrow S$ and functions $f_{1}, \ldots, f_{n}: S \rightarrow \mathbb{R}$. If the triples $\left\langle S, \tau, f_{i}\right\rangle$ are homomesic, then for a function $f$ which is a linear combination of the $f_{i} s,\left\langle S, \tau, f_{i}\right\rangle$ is homomesic.

The following theorem is a result of Propp and Roby [14]:

Theorem 2.2 ([14]). Consider $f: J\left(\mathcal{Q}_{a, b}\right) \rightarrow \mathbb{R}$ defined as follows: for all $I \in \mathcal{Q}_{a, b}$, $f(I)=|I|$. Let $\partial, \Phi: J\left(\mathcal{Q}_{a, b}\right) \rightarrow J\left(\mathcal{Q}_{a, b}\right)$ be the rowmotion and promotion operation. The triples $\left\langle J\left(\mathcal{Q}_{a, b}\right), \partial, f\right\rangle$ and $\left\langle J\left(\mathcal{Q}_{a, b}\right), \Phi, f\right\rangle$ exhibit homomesy.

We now present definition of comotion, which generalizes rowmotion and promotion. Later in this paper, we generalize Theorems 2.2 and 2.1 for comotion. 
Definition 2.9. For any permutation $\nu$ of $[a]$, we define the action comotion, $\mathcal{T}_{\nu}$ : $J(\mathcal{P}) \rightarrow J(\mathcal{P})$, for any $I \in J(\mathcal{P})$ as follows:

$$
\mathcal{T}_{\nu}(I)=\sigma_{C_{\nu(a)}} \circ \sigma_{C_{\nu(a-1)}} \circ \cdots \circ \sigma_{C_{\nu(1)}}(I)
$$

Remark 2. Note that by applying Proposition 2.1 inductively on the posets $\mathcal{Q}_{a, b}, \mathcal{U}_{a}$ and $\mathcal{L}_{a}$, the action of promotion coincides with $\mathcal{T}_{(a, a-1, \ldots 1)}$ and rowmotion coincides with $\mathcal{T}_{(1,2, \ldots a)}$.

We are now ready to state the main theorems of this paper, which are Theorems 2.3, 2.4, 2.5. We leave the proof of these theorems to Sections 3 and 4 . Before stating the main results we need to define the following functions which constitute building blocks of the functions for which we observe and prove homomesy:

Definition 2.10. Let $C_{i}$ be a column in $\mathcal{Q}_{a, b}, \mathcal{L}_{a}$ or $\mathcal{U}_{a}$. For $1 \leqslant i \leqslant a$ and $1 \leqslant j \leqslant b$, we define $g_{i, j}, s_{i, j}, d_{i, j}$, and $s_{j}$ and $\kappa_{j}$ for $I \in J(\mathcal{P})$ as follows:

- For any $1 \leqslant i \leqslant a$ and $1 \leqslant j \leqslant b$,

$$
g_{i, j}= \begin{cases}1, & \text { if }\left|C_{i}(I)\right|=j \\ 0, & \text { otherwise }\end{cases}
$$

- For any $1 \leqslant i \leqslant a$ and $1 \leqslant j \leqslant b$,

$$
s_{i, j}= \begin{cases}1 & \text { if }\left|C_{i}(I)\right|+i=j \\ 0 & \text { otherwise }\end{cases}
$$

- For any $1 \leqslant i \leqslant a$ and $1 \leqslant j \leqslant b$,

$$
d_{i, j}=g_{i, j}-g_{a+1-i, b-j} .
$$

- For any $1 \leqslant j \leqslant b$,

$$
s_{j}=\sum_{i=1}^{a} s_{i, j}
$$

- For any $1 \leqslant j \leqslant b$,

$$
\kappa_{j}=\sum_{i=1}^{a} g_{i, j} .
$$

In Theorems 2.3, 2.4, and 2.5 and Corollaries 2.1, 2.2, and 2.3, let $a$ be an arbitrary natural number and $\nu$ a permutation on $[a]$, and $\mathcal{T}_{\nu}$ as in Definition 2.9:

Theorem 2.3 (Homomesy in $\left.\boldsymbol{J}\left(\mathcal{Q}_{a, b}\right)\right)$. 1. The orbit structure of $\mathcal{T}_{\nu}$ on $J\left(\mathcal{Q}_{a, b}\right)$ is independent of choice of $\nu$. 
2. For any $1 \leqslant i \leqslant a$ and $1 \leqslant j \leqslant b$, the following triples are homomesic.

(a) $\left\langle\mathcal{T}_{\nu}, J\left(\mathcal{Q}_{a, b}\right), d_{i, j}\right\rangle$

(b) $\left\langle\mathcal{T}_{\nu}, J\left(\mathcal{Q}_{a, b}\right), s_{j}\right\rangle$.

Theorem 2.3 introduces a new family of permutations having the same orbit structure as $\Phi$ and $\partial$; hence, it generalizes Theorem 2.1. By Remark 2 any linear combination of the above functions is also homomesic, thus, Theorem 2.3 also generalizes Theorem 2.2 by introducing a wide class of permutations and statistics whose triples with $J\left(\mathcal{Q}_{a, b}\right)$ exhibit homomesy.

The proof of Theorem 2.3 is involved; we skip it in this section and will present it later in the paper. We will provide a roadmap to the proof after stating the following two theorems about homomesy in $J\left(\mathcal{U}_{a}\right)$ and $J\left(\mathcal{L}_{a}\right)$.

Theorem 2.4 (Homomesy in $\boldsymbol{J}\left(\mathcal{U}_{\boldsymbol{a}}\right)$ ). For each $i \in[2 a]$ let $[i, 2 a]=\{i, i+1, \ldots, 2 a\}$ and $f:[2 a] \rightarrow \mathbb{R}$ be a function having the same average in all $[i, 2 a]$ where $i$ is odd. Let $g: J\left(\mathcal{U}_{a}\right) \rightarrow \mathbb{R}$ be defined for any $I$ in $J\left(\mathcal{U}_{a}\right)$ as: $g(I)=\sum_{i=1}^{a} f\left(\left|C_{i}(I)\right|+2 i+1\right)$. Then, the triple $\left\langle J\left(\mathcal{U}_{a}\right), \mathcal{T}_{\nu}, g\right\rangle$ exhibits homomesy.

Theorem 2.5 (Homomesy in $\boldsymbol{J}\left(\mathcal{L}_{a}\right)$ ). 1. The orbit structure of $\mathcal{T}_{\nu}$ on $J\left(\mathcal{L}_{a}\right)$ is independent of choice of $\nu$.

2. For any $1 \leqslant j \leqslant a$, the triple $\left\langle J\left(\mathcal{L}_{a}\right), \mathcal{T}_{\nu}, \kappa_{j}\right\rangle$ exhibits homomesy.

The proofs of Theorem 2.3, 2.4, and 2.5 are postponed to the next sections. The heart of these proofs is the correspondence between comotion and a function which we define in Section 3 called winching (See Definition 3.1). We define three variations of winching on sequences of increasing numbers which correspond to comotion in $J(\mathcal{Q}), J(\mathcal{U})$, and $J(\mathcal{L})$.

A detailed presentation of these correspondences, together with formal definitions is given in Section 3. After introducing these definitions, we show that there is a natural equivariant bijection between the set of order ideals under comotion and the set of increasing sequences under winching. These correspondences simplify our theorems, and in fact in Section 3 we have Theorems 3.1, 3.2, and 3.3 which are in the context of winching and equivalent to Theorems 2.3, 2.4, and 2.5 of this section. Finally, in Section 4, by proving the theorems of Section 3 we complete the proofs of all the theorems in this paper.

We continue this section by employing the above theorems to find homomesy of some well known functions in the orbit structure produced by comotion in $J\left(\mathcal{Q}_{a, b}\right), J\left(\mathcal{U}_{a}\right)$, and $J\left(\mathcal{L}_{a}\right)$. For example one important function also studied by [14] is size of an order ideal, i.e. $s z(I)=|I|$. Here is a list of functions we consider:

1. Size. Let $s z$ be the size of an order ideal, i.e. $s z: J(\mathcal{P}) \rightarrow \mathbb{R} ; s z(I)=|I|$. 
2. Central antisymmetry. Let $x \in[a] \times[b]$. The antipodal point of $x$ (or its antipode) is $y$, if $x=(i, j)$ and $y=(a-i+1, b-j+1)$. We denote the antipodal point of $x$ by $A(x)$. For $I \in J(\mathcal{P})$ and $x \in[a] \times[b]$, we define the characteristic function $\mathcal{I}_{I}(x):[a] \times[b] \rightarrow\{0,1\}$ as follows:

$$
\mathcal{I}_{I}(x)= \begin{cases}1 & \text { if } x \in \mathcal{I} \\ 0 & \text { otherwise }\end{cases}
$$

For any arbitrary $x \in[a] \times[b]$, let $\mathcal{C} \mathcal{A}_{x}: J\left(\mathcal{Q}_{a, b}\right) \rightarrow\{0,1,-1\}$ be given by $\mathcal{C} \mathcal{A}_{x}(I)=$ $\mathcal{I}_{I}(x)-\left(1-\mathcal{I}_{I}(A(x))\right.$. We call $\mathcal{C} \mathcal{A}_{x}$ the central antisymmetry function with respect to $x$. The central antisymmetry function characterises the presence of one and only one of $x$ and its antipode in an order ideal.

3. Rank-alternating cardinality. Consider an arbitrary $I \in J(\mathcal{P})$. We denote the rank-alternating cardinality of $I$ by $\mathcal{R} \mathcal{A C}(I)$ and we define it as $\mathcal{R} \mathcal{A C}(I)=$ $\sum_{(i, j) \in I}(-1)^{i+j}$.

For any choice of $\nu$, the following homomesy results are concluded from Theorems $2.3,2.4$, and 2.5, and the fact that any linear combination of homomesic functions is homomesic (Remark 2):

Corollary 2.1. Let $\mathcal{P}$ be $\mathcal{Q}_{a, b}$ or $\mathcal{L}_{a}$. The triple $\left\langle J(\mathcal{P}), \mathcal{T}_{\nu}\right.$, sz $\rangle$ is homomesic.

Proof. For $\mathcal{P}=\mathcal{Q}_{a, b}, s z=\sum_{i=1}^{a} i s_{i}-a(a+1) / 2$. For $\mathcal{P}=\mathcal{L}_{a}, s z=\sum_{i=1}^{a} i s_{i}$. In both cases $s z$ is a linear combination of $s_{i}$. So, using Theorems 2.3 and 2.5 we will have the result.

Corollary 2.2. The triple $\left\langle J\left(\mathcal{Q}_{a, b}, \mathcal{T}_{\nu}, \mathcal{C} \mathcal{A}\right)\right\rangle$ exhibits homomesy.

Proof. Consider arbitrary $I \in \mathcal{Q}_{a, b}$ and $x=\left(x_{1}, x_{2}\right) \in[a] \times[b]$. Then $I_{\mathcal{I}}(x)=1$ if and only if $\left(x_{1}, x_{2}\right) \in \mathcal{I}$ which is equivalent to $\left|C_{x_{1}}(I)\right| \geqslant x_{2}$. Thus,

$$
I_{\mathcal{I}}(x)=\sum_{j=x_{2}}^{b} g_{x_{1}, j} .
$$

Similarly, $1-\mathcal{I}_{I}(A(x))=1$ implies $\left(a-x_{1}+1, b-x_{2}+1\right) \notin I$ meaning $\left|C_{a-x_{1}+1}(I)\right|<$ $b-x_{2}+1$ or equivalently $\left|C_{a-x_{1}+1}(I)\right| \leqslant b-x_{2}$. Therefore we have:

$$
1-\mathcal{I}_{I}(A(x))=\sum_{j=0}^{b-x_{2}} g_{a-x_{1}+1, j}=\sum_{j=x_{2}}^{b} g_{a-x_{1}+1, b-j} .
$$

By Equations 8 and 9, we have $\mathcal{C} \mathcal{A}_{x}(\mathcal{I})=\sum_{j=x_{2}}^{b} g_{x_{1}, j}-g_{a-x_{1}+1, b-j}$. Employing Theorem 2.3 we deduce that $\mathcal{C} \mathcal{A}_{x}$ is 0 -mesic for any arbitrary $x \in[a] \times[b]$.

Corollary 2.3. Let $\mathcal{P}$ be one of $\mathcal{Q}_{a, b}$ or $\mathcal{U}_{a}$. The triple $\left\langle J(\mathcal{P}), \mathcal{T}_{\nu}, \mathcal{R} \mathcal{A C}\right\rangle$ is homomesic. 
Proof. We will first consider the case when $I \in J\left(\mathcal{Q}_{a, b}\right)$. In this case we have:

$$
\begin{aligned}
2 \mathcal{R} \mathcal{A C}(I) & =\sum_{x=(i, j) \in \mathcal{P}}(-1)^{i+j} \mathcal{I}_{I}(x)=\sum_{x=(i, j)}(-1)^{i+j} \mathcal{I}_{I}(x)+\sum_{x=(i, j)}(-1)^{i+j} \mathcal{I}_{I}(x) \\
& =\sum_{x=(i, j) \in \mathcal{X}}(-1)^{i+j} \mathcal{I}_{I}(x)+(-1)^{2 a-(i+j)+2} \mathcal{I}_{I}(A(x)) \\
& =\sum_{x=(i, j) \in \mathcal{X}}(-1)^{i+j} \mathcal{C} \mathcal{A}_{x}(I)+1 .
\end{aligned}
$$

In the case where $I \in J\left(\mathcal{U}_{a}\right)$ we have:

$$
\mathcal{R} \mathcal{A C}(I)=(-1)^{a+1} \sum_{i ;\left|C_{i}\right| \text { is odd }} 1 .
$$

We define the function $f: \mathbb{N} \rightarrow\{0,1\}$ as follows: $f(x)=1$ if and only if $x$ odd, and $f(x)=0$ otherwise. For any odd $i$, the average of $f$ in $[i, 2 a]$ is equal to $1 / 2$. Therefore, the corollary is concluded from Theorem 2.4.

\section{Comotion, winching and their correspondence}

In the previous section, we defined the action of comotion on the set of order ideals of a poset. In this section, we define winching and show a correspondence between winching on increasing sequences and comotion on $J\left(\mathcal{Q}_{a, b}\right)$. Then, we define winching with lower bounds and winching with zeros. The former corresponds to comotion on $J\left(\mathcal{U}_{a}\right)$ and the later corresponds to comotion on $J\left(\mathcal{L}_{a}\right)$.

Definition 3.1. Let $S_{k, m}$ be the set of all $k$-tuples $x=\left(x_{1}, \ldots, x_{k}\right)$ satisfying $0<x_{1}<$ $x_{2}<\cdots<x_{k}<m+1$. We define the map $W_{i}: S_{k, m} \rightarrow S_{k, m}$, called winching on index $i$, by $W_{i}(x)=y=\left(y_{1}, y_{2}, \ldots, y_{k}\right)$, where $y_{j}=x_{j}$ for $i \neq j$, and

$$
y_{i}= \begin{cases}x_{i}+1, & \text { if } x_{i}+1<x_{i+1} . \\ x_{i-1}+1, & \text { otherwise. }\end{cases}
$$

We assume that always $x_{0}=0$ and $x_{k+1}=m+1$.

For arbitrary $\nu=\left(\nu_{1}, \nu_{2}, \ldots, \nu_{k}\right)$ a permutation of $[k]$ we define $W_{\nu}$ to be the function $W_{\nu_{k}} \circ W_{\nu_{k-1}} \circ \cdots \circ W_{\nu_{1}}$.

Example 3.1. Let $\nu=(2,3,1,4)$ and $x \in S_{4,7}$ be $x=(2,3,5,7)$. Then, $W_{\nu}(x)=$ $W_{4} \circ W_{1} \circ W_{3} \circ W_{2}(2,3,5,7)=W_{4} \circ W_{1} \circ W_{3}(2,4,5,7)=W_{4} \circ W_{1}(3,4,6,7)=W_{4}(1,4,6,7)=$ $(1,4,6,7)$.

We now show that winching is equivalent to toggling by columns on $J\left(\mathcal{Q}_{a, b}\right)$.

Lemma 3.1. Let $\alpha: J\left(\mathcal{Q}_{a, b}\right) \rightarrow S_{a, a+b}$ be defined as follows: $\alpha(I)=\left(\alpha_{1}, \ldots \alpha_{a}\right)$, and for any $1 \leqslant j \leqslant a$, we have $\alpha_{j}(I)=\left|C_{a+1-j}(I)\right|+j$. For any $I \in J\left(\mathcal{Q}_{a, b}\right), \alpha\left(\sigma_{C_{j}}(I)\right)=$ $W_{j}(\alpha(I))$. 
Proof. Consider $I \in J\left(\mathcal{Q}_{a, b}\right)$. For any $j_{1}<j_{2}$, since $\left|C_{j_{1}}(I)\right| \geqslant\left|C_{j_{2}}(I)\right|, \alpha(I)$ is an increasing sequence.

Let $C_{j}$ be $\left\{v_{1}, v_{2}, \ldots v_{b}\right\}$, where $v_{i}=(j, i)$, and assume $\left|C_{j}(I)\right|=l$. We have, $n>l+1$, $\sigma_{v_{n}}(I)=I$, and for $n=l+1, \sigma_{v_{n}}(I)=I \cup\left\{v_{n}\right\}$ if and only if $\left|C_{j-1}\right| \geqslant l+1$. Also, for $n<l, \sigma_{v_{n}}(I)=I-\left\{v_{n}\right\}$ if and only if $\left|C_{j+1}(I)\right| \leqslant n-1$. For boundary cases, we assume $\left|C_{0}\right|=b$ and $\left|C_{b}\right|=0$. Letting $K=\sigma_{C_{j}}(I)$ we have,

$$
C_{j}(K)= \begin{cases}C_{j}(I) \cup\left\{v_{l+1}\right\}, & \text { if }\left|C_{j-1}(I)\right| \geqslant l+1 . \\ C_{j}(I)-\left\{v_{l}, v_{l-1}, \ldots, v_{p+1}\right\}\left(p=\left|C_{j+1}(I)\right|\right), & \text { otherwise. }\end{cases}
$$

Equivalently,

$$
\left|C_{j}(K)\right|+a+1-j= \begin{cases}l+1+a+1-j, & \text { if }\left|C_{j-1}(I)\right|+a-j+2 \geqslant l+1+a-j+2 . \\ \left|C_{j+1}(I)\right|+a+1-j, & \text { otherwise. }\end{cases}
$$

Thus we have,

$$
\begin{aligned}
\alpha_{a+1-j}\left(\sigma_{C_{j}}(I)\right) & = \begin{cases}\alpha_{a-j+1}(I)+1, & \text { if } \alpha_{a-j+2}(I)>\alpha_{a-j+1}(I)+1 . \\
\alpha_{a-j}(I)+1, & \text { otherwise. }\end{cases} \\
& =W_{a+1-j}(\alpha(I)) .
\end{aligned}
$$

Thus, $\alpha\left(\sigma_{C_{j}}(I)\right)=W_{j}(\alpha(I))$.

Example 3.2. Figure 2 illustrates an application of Lemma 3.1 to an order ideal in $J\left(\mathcal{Q}_{4,5}\right)$. Note that as Lemma 3.1 suggests we have: $I_{2}=\sigma_{C_{3}}\left(I_{1}\right)$ and $I_{3}=\sigma_{C_{4}}\left(I_{2}\right)$. Equivalently we have: $W_{3}\left(\alpha\left(I_{1}\right)\right)=\alpha\left(I_{2}\right)$ and $W_{4}\left(\alpha\left(I_{2}\right)\right)=\alpha\left(I_{3}\right)$.

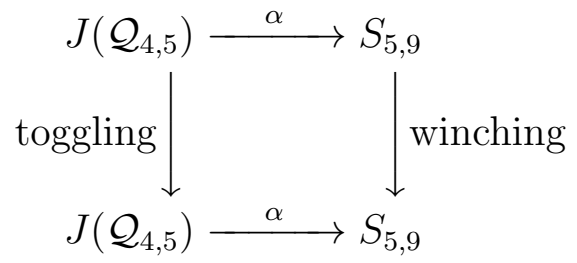

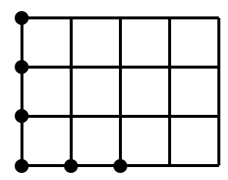

(a) $\alpha\left(I_{1}\right)=(1,2,4,5,9)$

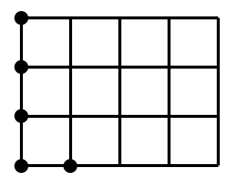

$\underset{\text { toggling on }}{\stackrel{\text { column } 4}{\longrightarrow}}$

(b) $\alpha\left(I_{2}\right)=(1,2,3,5,9)$

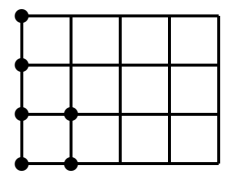

(c) $\alpha\left(I_{3}\right)=(1,2,3,6,9)$

Figure 2: The correspondence between toggling columns and winching an index 
In what follows we define two variations of winching: winching with lower bounds and winching with zeors. Then, we define two equivariant bijections from $J\left(\mathcal{U}_{a}\right)$ and $J\left(\mathcal{L}_{a}\right)$ to sequences of numbers. Finally, we show in Lemmas 3.2 and 3.3 that these bijections map toggling to winching with lower bounds in $J\left(\mathcal{U}_{a}\right)$ and to winching with zeros in $J\left(\mathcal{L}_{a}\right)$ (see Figure 3).
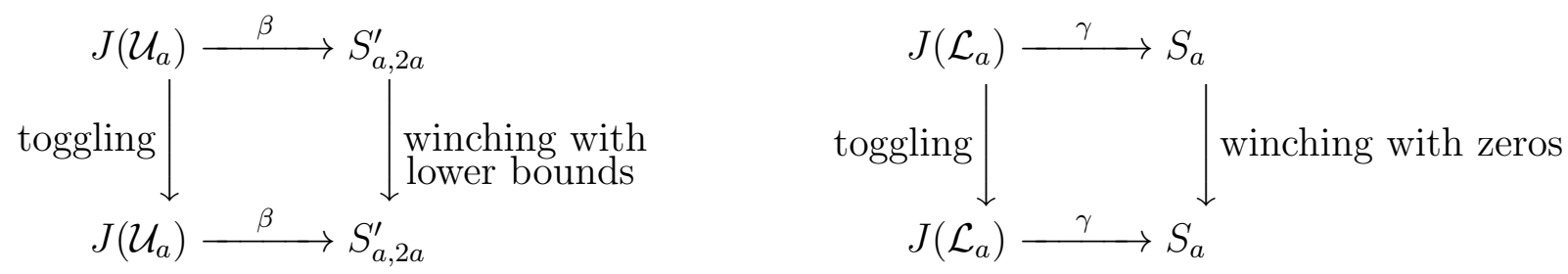

Figure 3: Equivariant bijections of Lemma 3.2 and Lemma 3.3.

The following variation of winching is called winching with lower bounds, and we show in Lemma 3.2 that it corresponds to comotion on $J\left(\mathcal{U}_{a}\right)$.

Definition 3.2. Consider the sequence of lower bounds $l=\left(l_{1}, \ldots, l_{k}\right), 0<l_{1}<\cdots<$ $l_{k}<m+1$ and $S_{k, m}^{\prime}=\left\{\left(x_{1}, x_{2}, \ldots x_{k}\right) \in S_{k, m} \mid x_{i} \geqslant l_{i}\right\}$, where $S_{k, m}$ is the set defined in Definition 3.1. For any index $i \in[k]$ and $w \in S_{k, m}^{\prime}$, we define the map $\underline{W}_{i}^{l}: S_{k, m}^{\prime} \rightarrow S_{k, m}^{\prime}$ called winching with lower bounds $l$ on index $i$ by

$$
\underline{W}_{i}^{l}(w)=\max \left\{W_{i}(w), l_{i}\right\}
$$

For any arbitrary permutation $\nu$ on $[a], I \in J\left(\mathcal{U}_{a}\right)$, and $x \in S_{a, 2 a}$, let the lower bounds be $l=(1,3, \ldots, 2 a-1)$. We define $\underline{W}_{\nu}^{l}(x)=\underline{W}_{\nu(a)}^{l} \circ \underline{W}_{\nu(a-1)}^{l} \circ \cdots \circ \underline{W}_{\nu(1)}^{l}(x)$.

Lemma 3.2. Let $\beta: J\left(\mathcal{U}_{a}\right) \rightarrow S_{a, 2 a}^{\prime}$ be defined as follows: $\beta(I)=\left(\beta_{1}, \ldots, \beta_{a}\right)$ and for any $1 \leqslant j \leqslant a, \beta_{j}(I)=\left|C_{a+1-j}(I)\right|+2 j-1$. For any $I \in J\left(\mathcal{U}_{a}\right)$ we have, $\beta\left(\sigma_{C_{j}}(I)\right)=$ $\underline{W}_{j}^{l}(\beta(I))$, where $l=(1,3,5, \ldots, 2 a-1)$.

Proof. Considering the order ideal $I \in J\left(\mathcal{Q}_{a}\right), I^{\prime}=I \cup\left(\mathcal{Q}_{a, a}-\mathcal{U}_{a}\right)$, we will have, $\beta(I)=$ $\alpha\left(I^{\prime}\right)$. Hence, $\beta$ is an increasing sequence. Since $\sigma_{C_{j}}(I)=\sigma_{C_{j}}\left(I^{\prime}\right)-\left(\mathcal{Q}_{a, a}-\mathcal{U}_{a}\right)$ we have,

$$
\begin{aligned}
\beta_{j}\left(\sigma_{C_{a+j-1}}(I)\right) & =\left|\sigma_{C_{a+j-1}}(I)\right|+2 j-1=\left|\sigma_{C_{a+j-1}}\left(I^{\prime}\right)-\left(\mathcal{Q}_{a, a}-\mathcal{U}_{a}\right)\right|+2 j-1 \\
& =\max \left\{\left|\sigma_{C_{a+j-1}}\left(I^{\prime}\right)\right|-j+1,0\right\}+2 j-1=\max \left\{\left|\sigma_{C_{a+j-1}}\left(I^{\prime}\right)\right|+j, 2 j-1\right\} \\
& =\max \left\{\left(W_{j}\left(\alpha\left(I^{\prime}\right)\right)\right)_{j}, 2 j-1\right\}=\max \left\{\left(W_{j}(\beta(I))\right)_{j}, 2 j-1\right\} .
\end{aligned}
$$

Thus, taking $l=(1,3,5, \ldots, 2 a-1)$, we conclude that $\beta\left(\sigma_{C_{j}}(I)\right)=\underline{W}_{j}^{l}(\beta(I))$. 
The following variation of winching is called winching with zeros, and we show in Lemma 3.3 that it corresponds to comotion on $J\left(\mathcal{L}_{a}\right)$.

Definition 3.3. Let $S_{n}$ be the set of all increasing sequences $x=\left(x_{1}, \ldots, x_{n}\right)$ of numbers in $[n]^{+}=\{0,1, \ldots, n\}$ such that for any $1 \leqslant i \leqslant n-1, x_{i}<x_{i+1}$ or $x_{i}=x_{i+1}=0$. We define the map $\mathrm{WZ}_{i}: S_{n} \rightarrow S_{n}$, called winching with zeros on index $i$ to be

$$
\mathrm{WZ}_{i}(x)= \begin{cases}x_{i}+1 & \text { if } x_{i}+1<\min \left\{x_{i+1}, n+1\right\} \\ x_{i-1}+1 & \text { if } 1<i \text { and } 0<x_{i-1} \\ 0 & \text { otherwise }\end{cases}
$$

Lemma 3.3. Let $\gamma: J\left(\mathcal{L}_{a}\right) \rightarrow S_{a}$ be defined as follows: $\gamma(I)=\left(\gamma_{1}, \gamma_{2}, \ldots, \gamma_{a}\right)$ and for any $1 \leqslant j \leqslant a, \gamma_{j}(I)=\left|C_{a+1-j}(I)\right|$. For any $I \in J\left(\mathcal{L}_{a}\right)$ we have $\gamma\left(\sigma_{C_{j}}(I)\right)=W Z_{j}(\gamma(I))$.

Proof. Consider arbitrary $I \in J\left(\mathcal{L}_{a}\right)$. For any $j_{1}<j_{2}$, we have $\left|C_{j_{1}}(I)\right|>\left|C_{j_{2}}(I)\right|$. Thus, $\gamma$ is an increasing sequence. Let $C_{j}=\left\{v_{j}, v_{j+1}, \ldots v_{a}\right\}$ where for $j \leqslant i \leqslant a, v_{i}=(j, i)$. Assume $\left|C_{j}(I)\right|=l$, which means $C_{j}(I)=\left\{v_{j}, v_{j+1}, \ldots v_{j+l-1}\right\}$. For $n>j+l, \sigma_{v_{n}}(I)=I$. We have three cases: if $n=j+l$, we will have $\sigma_{v_{n}}(I)=I \cup\left\{v_{n}\right\}$ if and only $(j-1, j+l) \in I$, that is, $\left|C_{j-1}(I)\right|>l+1$. If $C_{j+1}(I)=0, \sigma_{C_{j}}(I)=I-C_{j}(I)$. And if $\sigma_{C_{j}}(I)>0$, then $\sigma_{C_{j}}(I)=I-\left\{v_{k+1}, \ldots, v_{j+l-1}\right\}$, where $k=\left|C_{j+1}(I)\right|$. Letting $\sigma_{C_{j}}(I)=K$, we have:

$$
C_{j}(K)= \begin{cases}C_{j}(I) \cup\left\{v_{j+l}\right\}, & \text { if }\left|C_{j-1}(I)\right|>l+1 \\ \emptyset & \text { if }\left|C_{j-1}(I)\right| \leqslant l+1 \&\left|C_{j+1}(I)\right|=0 \\ C_{j}(I)-\left\{v_{k+1}, v_{k+2}, \ldots, v_{j+l-1}\right\}, & \text { otherwise. } \\ \quad, \text { where } k=\left|C_{j+1}(I)\right|>0 & \end{cases}
$$

which implies,

$$
\left|C_{j}(K)\right|= \begin{cases}l+1, & \text { if }\left|C_{j-1}(I)\right|>l+1 \\ 0 & \text { if }\left|C_{j-1}(I)\right| \leqslant l+1 \&\left|C_{j+1}(I)\right|=0 \\ k+1, \text { where } k=\left|C_{j+1}(I)\right|>0 & \text { otherwise. }\end{cases}
$$

and thus,

$$
\gamma_{j}(K)= \begin{cases}\gamma_{j}(I)+1, & \text { if } \gamma_{j+1}(I)>l+1 \\ 0 & \text { if } \gamma_{j+1}(I) \leqslant l+1 \& \gamma_{j-1}(I)=0 \\ \gamma_{j-1}+1, & \text { otherwise, }\end{cases}
$$

We thus conclude $\gamma_{a+1-j}\left(\sigma_{C_{j}}(I)\right)=W Z_{a+1-j}(\gamma(I))$.

Since comotion is a sequential composition of toggles applied to different columns, for any choice of $\nu$ we have the following corollaries:

Corollary 3.1. The bijection $\alpha$ introduced in Lemma 3.1 satisfies the following property: $\alpha\left(\mathcal{T}_{\nu}(I)\right)=W_{\nu}(\alpha(I))$. 
Corollary 3.2. The bijection $\beta$ introduced in Lemma 3.2 satisfies the following property: $\beta\left(\mathcal{T}_{\nu}(I)\right)=\underline{W}_{\nu}^{l}(\beta(I))$.

Corollary 3.3. The bijection $\gamma$ introduced in Lemma 3.3 satisfies the following property: $\gamma\left(\mathcal{T}_{\nu}(I)\right)=W Z_{\nu}(\gamma(I))$.

We now proceed to the theorems of this section, which discuss the occurrence of homomesy in the winching setting and are in correspondence with Theorems 2.3, 2.4 and 2.5 stated in the previous section.

We consider the following functions:

Definition 3.4. - Let $g_{i, j}: S_{k, m} \rightarrow \mathbb{R}, 1 \leqslant i \leqslant k$ and $1 \leqslant j \leqslant m$ be defined as follows:

$$
g_{i, j}(x)= \begin{cases}1, & \text { if } x_{i}=j \\ 0, & \text { otherwise }\end{cases}
$$

- For an arbitrary $1 \leqslant j \leqslant m$, let $f_{j}: S_{k, m} \rightarrow \mathbb{R}$ be defined by:

$$
f_{j}(x)= \begin{cases}1, & \text { if } j \in x \\ 0, & \text { otherwise }\end{cases}
$$

In Theorems 3.1, 3.2 and $3.3 \nu$ is an arbitrary permutation on $[k]$ or $[n]$. The proofs of these theorems will be presented in Section 4:

Theorem 3.1 (Homomesy for winching). 1. $W_{\nu}^{m}(x)=x$ for all $x \in S_{k, m}$.

2. We observe homomesy in the following triples:

(a) For each $1 \leqslant i, j \leqslant k$, the function $d_{i, j}=g_{i, j}-g_{k+1-i, m+1-j}$ is zero-mesic.

(b) For any $1 \leqslant j \leqslant m$, the triple $\left\langle S_{k, m}, W_{\nu}, f_{j}\right\rangle$ is homomesic and the average of $f_{j}$ along $W_{\nu}$ orbits is $\mathrm{k} / \mathrm{m}$.

We will prove the above Theorem in Section 4.1 .

Remark 3 . The orbit structure that winching produces on the set $S_{k, m}$ is the same as the orbit structure for rotation acting on the set of 2-colored necklaces with $k$ white beads and $m-k$ black beads, and hence independent of choice of $\nu$. (The orbit structure of necklaces is a classical problem in combinatorics, and it is known as Pólya's Theorem [11].)

Proof of Theorem 2.3. Given the bijection in Corollary 2.1, Theorem 2.3 is concluded from Theorem 3.1. 


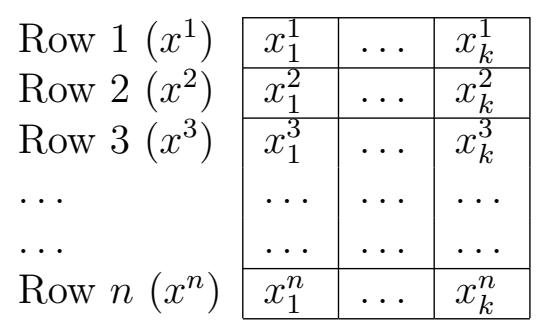

Figure 4: A tuple board.

Theorem 3.2 (Homomesy for winching with lower bounds). Consider $\underline{W}_{\nu}: S_{k, n}^{\prime} \rightarrow S_{k, n}^{\prime}$ with lower bounds $\left(l_{1}, l_{2}, \ldots, l_{k}\right)$. Let $[i, k+n]=i, i+1 \ldots k+n$ for an arbitrary $i \leqslant k+n$, and assume $f:[k+n] \rightarrow \mathbb{R}$ is a function that has the same average in all $\left[l_{i}, k+n\right]$. Let $g: S_{k, n}^{\prime} \rightarrow \mathbb{R}$ be defined as, $g(x)=\sum_{i=1}^{k} f\left(x_{i}\right)$. Then, the triple $\left\langle S_{k, n}^{\prime}, \underline{W}_{\nu}, g\right\rangle$ exhibits homomesy.

We will prove the above Theorem in Section 4.2.

Proof of Theorem 2.4. Given the bijection in Corollary 2.2, Theorem 2.4 is concluded from Theorem 3.2 .

Theorem 3.3 (Homomesy for winching with zeros). $\quad$ 1. $W Z_{\nu}^{2 n}(x)=x$ for all $x \in S_{n}$.

2. The triple $\left\langle S_{n}, W Z_{\nu}, f_{j}\right\rangle$ (defined in Definition 3.4) is homomesic. Furthermore, the average of $f_{j}$ along $W Z_{\nu}$-orbits is $\frac{1}{2}$.

We will prove the above theorem in Section 4.3.

Proof of Theorem 2.5. Given the bijection in Corollary 2.3, Theorem 2.5 is concluded from Theorem 3.3 .

\section{Proofs}

In this section we will prove Theorems 3.1, 3.2, and 3.3. The concepts of a tuple board and a snake (Definitions 4.1 and 4.2) play a key role in understanding the orbit structure and homomesy in winching.

For fixed $k$ and an arbitrary permutation on $[k]$, namely $\nu=\left(\nu_{1}, \nu_{2}, \ldots, \nu_{k}\right)$, let $\mathcal{F}_{\nu}$ be one of $W_{\nu}, \underline{W}_{\nu}$ or $W Z_{\nu}$. Let $S=S_{k}$ if $\mathcal{F}_{\nu}=W Z_{\nu}$ and $S=S_{k, m}$ otherwise. We define a tuple board as follows:

Definition 4.1. Let $x \in S$. A tuple board of $x$ is an $n \times k$ matrix whose rows are $x, \mathcal{F}_{\nu}(x), \mathcal{F}_{\nu}^{2}(x), \ldots$ (See Figure 4). Precisely, $T B(x)=\operatorname{transpose}\left(\left[x^{1}, x^{2}, \ldots, x^{n}\right]\right)$, where $T B(i, \cdot)=x^{i}=\left(x_{1}^{i}, \ldots, x_{k}^{i}\right), x^{i}=\mathcal{F}_{\nu}^{i-1}(x)$, and $\mathcal{F}_{\nu}{ }^{n+1}(x)=x$. 
Remark 4. Note that since $\mathcal{F}$ is a permutation, there is always an $n$ satisfying $\mathcal{F}_{\nu}{ }^{n+1}(x)=$ $x$. In fact, a tuple board contains an orbit of winching, thus it is convenient to think of it as a cylinder. When referring to row numbers in a tuple board they are understood modulo $n$.

Notice that any cell in a tuple board contains a number from the set $\{0,1,2, \ldots, m\}$. In any tuple board we partition the cells having non-zero elements to maximal sequences of adjacent cells of consecutive numbers. We call any of such partitions a snake. More precisely:

Definition 4.2. For an arbitrary $x \in S$, let $T B=T B(x)$ and $T=\{T B(i, j) \mid 1 \leqslant$ $i \leqslant n, 1 \leqslant j \leqslant k\}$. We define a snake $s=\left(s_{h}, s_{h+1}, \ldots, s_{t}\right)$ as follows: $s$ is a maximal sequence such that each $s_{i}$ is a cell in the tuple board having number $1 \leqslant i \leqslant n$ in it, and for any $i, s_{i}=\mathcal{M}\left(s_{i-1}\right)$, where $\mathcal{M}$ is defined as follows:

$$
\mathcal{M}(T(i, j))= \begin{cases}T(i+1, j) & \text { if } T(i+1, j)=T(i, j)+1 . \\ T(i, j+1) & \text { if } T(i, j+1)=T(i, j)+1, \quad T(i+1, j) \neq T(i, j)+1 \\ & \text { and } \nu(j)<\nu(j+1) . \\ T(i+1, j+1) & \text { if } T(i+1, j+1)=T(i, j)+1, T(i+1, j) \neq T(i, j)+1 \\ & \text { and } \nu(j)>\nu(j+1) .\end{cases}
$$

Definition 4.3. For a tuple board $T$, we define the function snake map, denoted $\mathcal{S}$, from the set of all snakes in $T$ to $\mathbb{N}^{k}$ as follows: any snake $s=\left(s_{h}, s_{h+1}, \ldots, s_{t}\right)$ is mapped to $\mathcal{S}(s)=\left(c_{1}, c_{2}, \ldots, c_{k}\right)$, where $c_{j}=|\{i \mid T(i, j) \in s\}|$. Moreover, in $s$ we call $h$ the head of $s$, and $t$ its tail, denoting them respectively by $\mathcal{H}(s)$ and $\mathcal{T}(s)$. The length of $s$ is equal to $\mathcal{T}(s)-\mathcal{H}(s)+1$. Two snakes are called consecutive if the submatrix in $T$ between their heads intersects no other snake. For two arbitrary snakes $s$ and $s^{\prime}$ in $T$, we say $s>s^{\prime}$ if the row number of $\mathcal{H}(s)$ in the tuple board is less than the row number of $\mathcal{H}\left(s^{\prime}\right)$.

Depending on the choice of $\mathcal{F}_{v}$, tuple boards will be filled with snakes differently. The proofs of Theorems 3.1, 3.2 and 3.3 are based on understanding the configuration of snakes in tuple boards. Figure 5 illustrates this by presenting three examples of snake configurations corresponding to winching, winching with lower bounds and winching with zeros. We formalize these observations in proofs of Theorems 3.1, 3.2 and 3.3 presented in the following subsections.

\subsection{Proof of Theorem 3.1}

In this subsection, we prove Theorem 3.1. There are two main observations about the placement of snakes in a tuple board corresponding to winching which construct our proof: In any winching tuple board (1) each snake has length $k$, its head is 1 and its tail is $k$, (2) the snake maps evolve through the left shift operator (Definition 4.4). We prove items (1) and (2) in Lemmas 4.1 and 4.2. 


\begin{tabular}{|l|l|l|l|l|}
$\ldots$ & $\ldots$ & $\ldots$ & $\ldots$ & $\ldots$ \\
\hline 1 & 2 & $?$ & $?$ & $?$ \\
\hline$?$ & 3 & $?$ & $?$ & $?$ \\
\hline$?$ & 4 & 5 & $?$ & $?$ \\
\hline$?$ & $?$ & 6 & $?$ & $?$ \\
\hline$?$ & $?$ & 7 & $?$ & $?$ \\
\hline$?$ & $?$ & $?$ & 8 & 9 \\
\hline$?$ & $?$ & $?$ & $?$ & 10 \\
\hline$\ldots$ & $\ldots$ & $\ldots$ & $\ldots$ & $\ldots$
\end{tabular}

A tuple board of $W_{\nu}$, $x \in S_{5,10}, \nu=(1,2,4,3,5)$. The snake map is $(1,3,3,1,2)$.

\begin{tabular}{|l|l|l|l|l|}
$\ldots$ & $\ldots$ & $\ldots$ & $\ldots$ & $\ldots$ \\
\hline$?$ & 4 & $?$ & $?$ & $?$ \\
\hline$?$ & $?$ & 5 & $?$ & $?$ \\
\hline$?$ & $?$ & 6 & $?$ & $?$ \\
\hline$?$ & $?$ & 7 & $?$ & $?$ \\
\hline$?$ & $?$ & 8 & 9 & 10 \\
\hline$?$ & $?$ & $?$ & $?$ & $?$ \\
\hline$\ldots$ & $\ldots$ & $\ldots$ & $\ldots$ & $\ldots$ \\
$\ldots$ & $\ldots$ & $\ldots$ & $\ldots$ & $\ldots$
\end{tabular}

A tuple board of $W_{\nu}$ with lower bounds $(2,4,6,7,8)$, $x \in S_{5,10}, \nu=(1,3,2,4,5)$. The snake map is $(0,1,4,1,1)$

\begin{tabular}{|l|l|l|l|l|}
$\ldots$ & $\ldots$ & $\ldots$ & $\ldots$ & $\ldots$ \\
$\ldots$ & $\ldots$ & $\ldots$ & $\ldots$ & $\ldots$ \\
\hline 0 & 1 & $?$ & $?$ & $?$ \\
\hline$?$ & $?$ & 2 & $?$ & $?$ \\
\hline$?$ & $?$ & 3 & 4 & 5 \\
\hline$?$ & $?$ & $?$ & $?$ & $?$ \\
\hline$?$ & $?$ & $?$ & $?$ & $?$ \\
\hline$\ldots$ & $\ldots$ & $\ldots$ & $\ldots$ & $\ldots$ \\
$\ldots$ & $\ldots$ & $\ldots$ & $\ldots$ & $\ldots$ \\
\hline
\end{tabular}

A tuple board of $W Z_{\nu}$, $x \in S_{5}, \nu=(1,3,2,4,5)$. The snake map is $(0,1,2,1,1)$.

Figure 5: Configurations of snake maps for different versions of winching.

In the rest of this subsection $T=T B(x)$ is a tuple board corresponding to winching and $x \in S_{k, m}$.

Lemma 4.1. Let $s$ be a snake in $T$. Then $\mathcal{H}(s)=1$ and $\mathcal{T}(s)=k$, thus the length of $s$ is $k$. Furthermore, for any two snakes $s$ and $s^{\prime}$ in $T$ either all $k$ cells they occupy in $T$ are the same or they share no common cell in $T$.

Proof. Consider $x$ and a snake $s$ in the tuple board $T=T B(x)=\left[x^{1}, \ldots, x^{n}\right]$. Assume $\mathcal{H}(s)=h \neq 1$ and that $s_{\mathcal{H}(s)}$ is in row $i$ and column $j$. We have $W_{\nu}\left(x^{i-1}\right)=x^{i}$. Let $y$ be equal to $W_{\nu(1)} \circ W_{\nu(2)} \circ \cdots \circ W_{\nu(j-1)}\left(x^{i-1}\right)$. By the definition of winching, if $x_{j}^{i-1} \neq h-1$ we will have $x_{j}^{i}=y_{j-1}+1$. If $\nu(j-1)<\nu(j)$ we have $y_{j-1}=x_{j-1}^{i}=h-1$. If $\nu(j-1)>\nu(j)$ we have $y_{j-1}=x_{j-1}^{i-1}=h-1$. This is in contradiction since a snake is defined as a maximal sequence of cells in the $T$ related by $\mathcal{M}$. Similarly we can show that $\mathcal{T}(s) \neq k$ is in contradiction with maximality of $s$.

To see that two different snakes are either the same or have no intersection, assume we have $s=\left(s_{1}, s_{2}, \ldots s_{k}\right)$ and $s^{\prime}=\left(s_{1}, s_{2}, \ldots, s_{k}\right)$. First assume $s_{\mathcal{H}(s)}=s_{\mathcal{H}\left(s^{\prime}\right)}^{\prime}$ let $l>1$ be the minimum index such that $s_{l} \neq s_{l}^{\prime}$. Consider the pair $i, j$ such that $T(i, j)=l$, $T(i+1, j)=l+1$ and also either $T(i, j+1)=l+1$ or $T(i+1, j+1)=l+1$. However, in this case by Definition 4.2 we have $s_{l}=s_{l}=T(i+1, j)$ which is a contradiction. Now assume $s_{\mathcal{H}(s)}=T(1, r), s_{\mathcal{H}\left(s^{\prime}\right)}^{\prime}=T\left(1, r^{\prime}\right)$ and without loss of generality assume that $r>r^{\prime}$ and $\nu=(1,2, \ldots k)$. Let $l>1$ be the minimum index such that $s_{l}=s_{l}^{\prime}=T(i, j)$. But this is contradiction because $l=j+1+(i-r)$ and $l=j+1+\left(i-r^{\prime}\right)$ which is only possible if $r=r^{\prime}$.

Definition 4.4. Let $H:[m]^{[k]} \rightarrow[m]^{[k]}$ be defined as follows: For $x=\left(x_{1}, \ldots, x_{k}\right)$, let $H(x)=y=\left(y_{1}, \ldots, y_{k}\right)$ where for any $1 \leqslant i<k, y_{i}=x_{i+1}$ and $y_{k}=x_{1}$. We call $H$ the left shift operator. 
In the next lemma we show that two consecutive snakes maps are related by the left shift operator. This is essential to the proof of Theorem 3.1.

Lemma 4.2. Consider an arbitrary tuple board $T$, and let $s<s^{\prime}$ be two consecutive snakes in it. Assume $c=\mathcal{S}(s)$ and $c^{\prime}=\mathcal{S}\left(s^{\prime}\right)$ are the two corresponding snake maps. We have $c=H\left(c^{\prime}\right)$.

Proof. Take $c=\left(c_{1}, c_{2}, \ldots c_{k}\right)$ and $c^{\prime}=\left(c_{1}^{\prime}, c_{2}^{\prime}, \ldots c_{k}^{\prime}\right)$. We first show that for any $2 \leqslant j \leqslant$ $k, c_{j-1}=c_{j}^{\prime}$. Then having that both $s$ and $s^{\prime}$ have length $k$ we also conclude $c_{k}=c_{1}^{\prime}$. For any arbitrary $2 \leqslant j \leqslant k$, using induction we show that if $f=\min \{i \mid T(i, j-1) \in s\}$, $l=\max \{i \mid T(i, j-1) \in s\}$, and $f^{\prime}=\min \left\{i \mid T(i, j) \in s^{\prime}\right\}, l^{\prime}=\max \left\{i \mid T(i, j) \in s^{\prime}\right\}$, then $f=f^{\prime}+1$ and $l=l^{\prime}+1$. For $j=2$ this is clear by the fact $s$ and $s^{\prime}$ have no intersection.

By the induction hypothesis assume that we have $c_{1}=c_{2}^{\prime}, c_{2}=c_{3}^{\prime}, \ldots, c_{j-1}=c_{j}^{\prime}$. We want to prove that $c_{j}=c_{j+1}^{\prime}$. Let $q$ and $q^{\prime}$ be, respectively, the length of $s$ truncated within columns 1 through $j$ and the length of $s^{\prime}$ truncated within columns 1 through $j-1$. From the inductive hypothesis, we conclude $q=q^{\prime}+c_{1}>q^{\prime}$. Thus if $c_{j}>c_{j+1}^{\prime}$ then either length of $s^{\prime}$ is less than length of $s$ which is a contradiction or $s$ and $s^{\prime}$ intersect which is also a contradiction. If $c_{j}<c_{j+1}^{\prime}$ then there is a gap between $s$ and $s^{\prime}$ but this is also a contradiction. Thus, we have $c_{j}=c_{j+1}^{\prime}$ (see Figure 6).

Corollary 4.1. There are exacly $k$ snakes covering a tuple board.

Proof. Since the left shift operator satisfies $H^{k}(c)=c$, the placements of the $i$ th snake and the $(i+k)$ th snake will be the same. Thus, the rows in the tuple board will be repeated after the $k$ th snake appears in the tuple board.

Proof of Theorem 3.1, Part 1. Consider an $n \times k$ tuple board $T$ such that $T=T B(x)$ and $x \in S_{k, m}$. Let's assume that $n \geqslant m$ (if $n<m$, append enough copies of $T$ to it until $n \geqslant m)$. Let $s^{1}$ be the snake that covers $T(1,1)$ and $s^{2}>s^{3}>s^{4}>\cdots>s^{k}$ be the rest of consecutive snakes covering $T$. Letting $\mathcal{S}\left(s^{1}\right)=c=\left(c_{1}, c_{2}, \ldots, c_{k}\right)$, by Lemma 4.2 we have $\mathcal{S}\left(s^{i}\right)=H^{i-1}(c)$. The numbers in the first column of $T$ will be: $x_{1}, x_{1}+1, \ldots, x_{1}+c_{1}-1,1,2, \ldots c_{2}, 1,2, \ldots, c_{3}, \ldots$ Since $\sum_{i=1}^{k} c_{i}=m$, the $(m+1)$ st number in the first column will be $x_{1}$. Similarly, for each column $i$, the $(m+1)$ st element will be $x_{i}$. Thus, $W^{m+1}(x)=x$.

Lemma 4.3. Let $T$ be an $m \times k$ tuple board. Consider the column $r: T_{r}=\{T(i, r)\}$. For any $1 \leqslant r \leqslant k$, there exists a one-to-one function $\mathcal{F}: T_{r} \rightarrow T_{k+1-r}$, satisfying $\mathcal{F}(x)=m+1-x$.

Proof. For any $r$, we construct a mapping from $\left\{\cup_{t=1}^{r} T_{t}\right\}$ to $\left\{\cup_{t=k-r+1}^{k} T_{t}\right\}$. Consider a number $x$ in $T_{r}$. Let it be the $l$ th element in $T_{r}$, covered by a snake having snake map $p=$ $\left(c_{1}, c_{2}, \ldots, c_{r}, \ldots, c_{k}\right)$. Consider the snake with snake map $p^{\prime}=\left(c_{r+1}, \ldots, c_{1}, c_{2}, \ldots, c_{r}\right)$. Let $y$ be the $\sum_{i=1}^{r-1}\left(c_{i}+l\right)$ th element from the end in this snake. Then $y=m+1-$ $\left(\sum_{i=1}^{r-1} c_{i}\right)+l=m+1-x$. Since $\left(\sum_{i=1}^{r-1} c_{i}\right)+l \leqslant \sum_{i=1}^{r} c_{i}, y$ will be lying in one of the columns $k, \ldots, k-r+1$. 


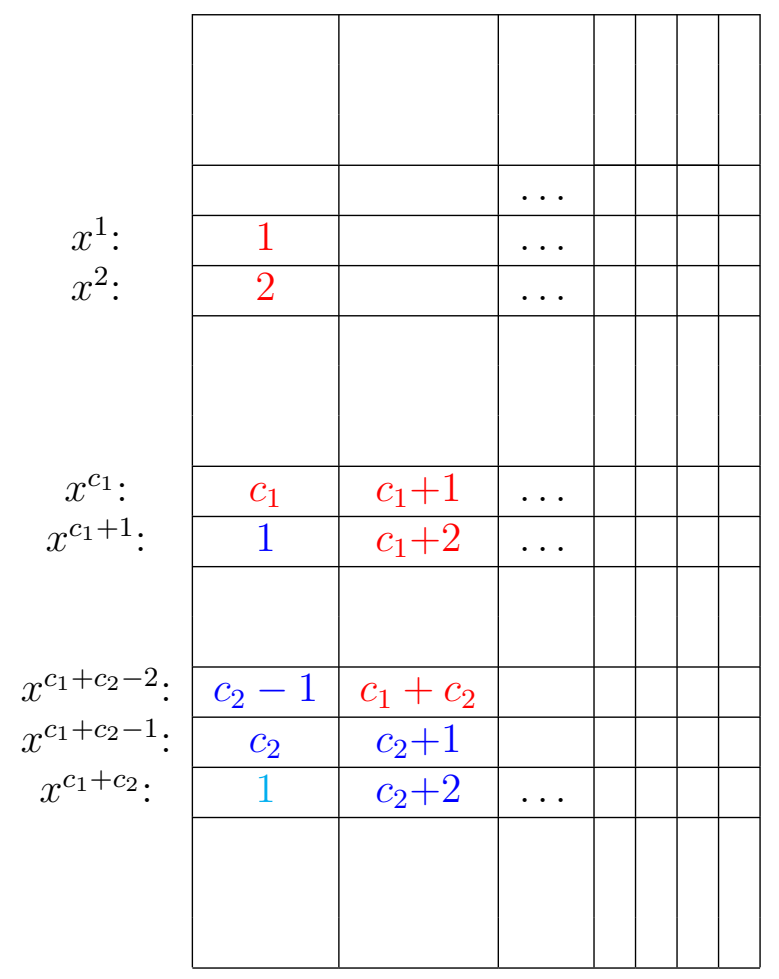

\begin{tabular}{|l|l|l|l|}
\hline 2 & 3 & 4 & 6 \\
\hline 1 & 2 & 5 & 7 \\
\hline 1 & 3 & 6 & 7 \\
\hline 2 & 4 & 5 & 6 \\
\hline 3 & 4 & 5 & 7 \\
\hline 1 & 2 & 6 & 7 \\
\hline 1 & 3 & 4 & 5 \\
\hline
\end{tabular}

Figure 6: Snakes in a tuple board of winching: the left hand side picture shows placement of two consecutive snakes in general form, the right hand side picture shows an example of a tuple board being covered by 4 consecutive snakes.

Having the above mapping, we know there is also a one-to-one mapping in $\left\{\cup_{t=1}^{r} T_{t}\right\} \rightarrow$ $\left\{\cup_{t=k-r+1}^{k} T_{t}\right\}$ and also in $\left\{\cup_{t=1}^{r-1} T_{t}\right\} \rightarrow\left\{\cup_{t=k-r}^{k} T_{t}\right\}$. Hence, there exists $\mathcal{F}: T_{r} \rightarrow T_{k+1-r}$ satisfying $\mathcal{F}(x)=m+1-x$.

Proof of Theorem 3.1, Part 2. Considering any $m \times k$ tuple board T, Corollary 4.1 shows that $T$ is totally covered by $k$ snakes. Therefore, each element $1 \leqslant i \leqslant m$ appears $k$ times in the tuple board and therefore the average of $f_{i}$ as defined in Definition 3.4 is independent of the orbit and equal to $\mathrm{k} / \mathrm{m}$.

Fix an arbitrary number $j$ and column $i$. Lemma 4.3 shows that the number of occurrences of number $j$ in column $i$ is equal to the number of occurrences of $m-j+1$ in column $k-i+1$ of $T$. Thus, $\sum_{\alpha=0}^{m-1} g_{i, j} W^{\alpha}(x)=\sum_{\alpha=0}^{m-1} g_{k-i+1, m-j+1}\left(W^{\alpha}(x)\right)$. In other words, for all $1 \leqslant i \leqslant k, 1 \leqslant j \leqslant m, g_{i, j}-g_{k-i+1, m-j+1}$ is zero-mesic in $W$-orbits of $S_{k, m}$.

\subsection{Proof of Theorem 3.2}

In this subsection we prove Theorem 3.2. Remember the definitions of tuple board, snake, snake map and the correspondence to the action of winching with lower bounds. 
Recall Lemma 4.1, and that if $s$ is a snake in a tuple board of winching then $\mathcal{H}(s)=1$ and $\mathcal{T}(s)=m$. In the following lemma we will show that if $s$ is a $\underline{W}_{\nu}^{l}$-snake with lower bounds $l=\left(l_{1}, l_{2}, \ldots, l_{k}\right)$, then $\mathcal{H}(s)$ is equal to some $l_{i}$ and $\mathcal{T}(s)=m$ (see Figure 5 ).

Lemma 4.4. For an arbitrary $x \in S_{k, m}$, consider a tuple cylinder $T(x)$ corresponding to application of the action of $\underline{W}_{\nu}^{l}$ with lower bounds $l=\left(l_{1}, l_{2}, \ldots, l_{k}\right)$. For any snake $s$ in this tuple board we have $\mathcal{H}(s) \in l$, and $\mathcal{T}(s)=m$.

Proof. Consider a snake $s$ in $T$. If $\mathcal{H}(s)$ is in column $i$ and $\mathcal{H}(s) \geqslant l_{i}$ then $s$ is not maximal. If $\mathcal{T}(s) \leqslant m$, then $s$ is not maximal either. Thus we have $\mathcal{H}(s) \in l$, and $\mathcal{T}(s)=m$.

Proof of Theorem 3.2. Any tuple cylinder corresponding to $\underline{W}_{\nu}^{l}$ can be partitioned into snakes. Any snake starts with some $l_{i} \in l$ and ends in $m$. Thus, if $f$ is a function having the same average on all the numbers contained in any snake, it has the same average over all the elements in the tuple cylinders. Therefore, we will have the result.

\subsection{Proof of Theorem 3.3}

In this section, we will prove Theorem 3.3. Consider $x \in S_{n}$ and the action of $W Z_{\nu}$ for some arbitrary permutation $\nu$ of $[n]$, and consider $T=T B(x)$. In what follows, we will prove Lemmas 4.5, 4.6, 4.7 which correspondingly show: In any tuple board of winching with zeros (1) any snake has length $n,(2)$ the snake maps evolve through a bijection called "crawling" (Definition 4.6), and in tuple board of winching with zeros there is one and only one row between heads of any two consecutive snakes. Finally, (3) crawling has orbit size $n$.

The proof of the following lemma is similar to the proof of Lemma 4.1, thus, we omit it.

Lemma 4.5. Let $s$ be a snake in a tuple board of winching with zeros. We have $\mathcal{H}(s)=1$ and $\mathcal{T}(s)=n$.

We now need to characterize the snake maps in winching with zeros. We first present the definition of crawl (Definition 4.6) and then we present Lemma 4.6.

Definition 4.5. Let $M_{n}$ be the set of all sequences $\left(c_{1}, c_{2}, \ldots, c_{n}\right)$ having an initial segment of all zeros and a nonzero segment $c_{k_{c}}, c_{k_{c}+1}, \ldots, c_{n}$ summing to $n$, that is, $\sum_{i=1}^{n} c_{i}=n$, and there is a $k_{c}$ such that $c_{i}=0$ if and only if $i<k_{c}$.

Definition 4.6. We define the action crawl $C: M_{n} \rightarrow M_{n}$ such that for any $c \in M_{n}$, $C(c)=c^{\prime}$ where for $1 \leqslant i \leqslant n-1$,

$$
c_{i}^{\prime}= \begin{cases}\max \left\{0, c_{i+1}-1\right\} & \text { If } c_{1}, \ldots, c_{i} \leqslant 1 \\ c_{i+1} & \text { otherwise. }\end{cases}
$$

, and $c_{n}^{\prime}=n-\sum_{i=1}^{n-1} c_{i}^{\prime}$. 


\begin{tabular}{|l|l|l|l|}
\hline 0 & 0 & 1 & 4 \\
\hline 0 & 0 & 2 & 3 \\
\hline 0 & 1 & 2 & 4 \\
\hline 0 & 0 & 3 & 4 \\
\hline 0 & 1 & 2 & 3 \\
\hline 0 & 0 & 0 & 4 \\
\hline 0 & 0 & 1 & 2 \\
\hline 0 & 0 & 0 & 3 \\
\hline
\end{tabular}

snake maps: $\quad(0,0,2,2)$

$(0,0,1,3)$

Figure 7: Snakes in a tuple board of winching with zeros: heads are located in alternating rows. The snake maps evolve through "crawling".

We now show that crawling actually captures the evolution of snakes in a tuple board $T$ corresponding to winching with zeros:

Lemma 4.6. Consider two consecutive snakes $s>s^{\prime}$ in $T$. If $\mathcal{H}(s)$ is in row $i$ of $T$ then $\mathcal{H}\left(s^{\prime}\right)$ is located in row $i+2$. Furthermore, let $c=\left(c_{1}, \ldots, c_{n}\right)$ be the snake map of $s$ and $c^{\prime}=\left(c_{1}^{\prime}, \ldots, c_{n}^{\prime}\right)$ be the snake map of $s^{\prime}$. We have $c^{\prime}=C(c)$.

Proof. Consider a snake $s$ whose head is at row $i$ in $T$ and assume that in its snake map, the initial segment $c_{1}, c_{2}, \ldots, c_{k_{0}}$ is all zeros, and this segment is followed by $c_{k_{0}+1}, \ldots, c_{k_{1}}$ all ones. These two segments are then followed by $c_{k_{1}+1}, \ldots$ all greater than 1 . This means that $x^{i}=\left(x_{1}, x_{2}, \ldots, x_{n}\right)$ and $x_{1}, x_{2}, \ldots, x_{k_{0}}=0$, for $i \leqslant k_{1}-k_{0} x_{i+k_{0}}=i$, and $x_{k_{1}+1} \geqslant k_{1}-k_{0}+1$. Thus applying winching with zeros to $x^{i}$, we take $x^{\prime}=x^{i+1}$. Thus we have $x^{i+1}=\left(x_{1}^{\prime}, x_{2}^{\prime}, \ldots, x_{n}^{\prime}\right)$ satisfying $x_{i}^{\prime}=0$ for any $i<k_{1}$ and $x_{k_{1}}^{\prime}=x_{k_{1}}+1$. Applying winching once again, and letting $x^{i+2}=x^{\prime \prime}$, we have $x^{i+2}=\left(x_{1}^{\prime \prime}, x_{2}^{\prime \prime}, \ldots, x_{n}^{\prime \prime}\right)$ satisfying $x_{i}^{\prime \prime}=0$ for any $i<k_{1}-1$ and $x_{k_{1}-1}^{\prime \prime}=1$. Thus, $\mathcal{H}\left(s^{\prime}\right)$ will be in row $i+2$ and in $c^{\prime}$ we will have: $c_{0}^{\prime}, c_{1}^{\prime}, \ldots, c_{k_{1}-1}^{\prime}=0$.

Note that in the action of crawling the initial segment which is all zeros and ones is mapped to a sequence of all zeros and the rest of the elements in the sequence evolve through the left shift operator. We have so far proved that the initial segment is mapped to all zeros. In remains to prove that $c_{k_{1}}^{\prime}=c_{k_{1}+1}-1$ and for any $i>k_{1}, c_{i}^{\prime}=c_{i+1}$.

Similar to proof of Lemma 4.2 we can conclude $c_{k_{1}}^{\prime}=c_{k_{1}+1}-1$ because the tuple board is covered by the snakes of length $n$, and that there is one row between $\mathcal{H}(s)$ and $\mathcal{H}\left(s^{\prime}\right)$. Since after this point there is no gap between the snakes we have: for any $i>k_{1}$, $c_{i}^{\prime}=c_{i+1}$. To complete the proof, note that $c_{n}$ and $c_{n}^{\prime}$ should always be such that the entire snake map sums to $n$, thus we have: $c_{n}^{\prime}=n-\sum_{i=1}^{n-1} c_{i}^{\prime}$ (see Figure 7).

The next lemma which is Lemma 4.7 completes the characterization of $W Z$-snakes. In particular it shows that the orbit size of crawl is $n$. The proof of this lemma is involved, and we prove it through a series of definitions and lemmas. (Definition 4.7, Lemma 4.8, Definition 4.8, Lemma 4.3 and Lemma 4.10.)

Here, after stating Lemma 4.7 we use it to prove Theorem 3.3. Then we finish this section by proving Lemma 4.7 . 
Lemma 4.7. For any $c \in M_{n}$ we have, $C^{n}(c)=c$.

Proof of Theorem 3.3 Part 1. From Lemma 4.6 we know that head of snakes are located in alternating rows. By Lemma 4.7 we know that each snake gets back to itself after $n$ crawls. Thus, in a tuple board of winching with zeros the 1 st and $2 n$th rows are identical, i.e. $W Z^{2 n}(x)=x$.

Proof of Theorem 3.3 Part 2. Consider a tuple board of winching with zeros. In the previous part of this theorem we proved that this board is a $2 n \times n$ board. In Lemma 4.6 we showed that head of snakes appear alternatively in rows. Thus, the number of snakes is $n$, and we conclude that half of the tuple board is filled with zeros. In addition, since there are $n$ snakes in any tuple board and in any snake $j$ appears once and only once, each nonzero number will appear $n$ times in the tuple board, that is, the average of $f_{j}=\frac{1}{2}$ for each $j$.

We now proceed to prove Lemma 4.7. To this end, we introduce two bijections in Definitions 4.7 and 4.8. In Lemma 4.3 we show that the bijection $\mathcal{F}$ (Definition 4.7) preserves the orbit structure of winching with zeros.

Definition 4.7. Consider the set $M_{n}$ from Definition 4.5. We define the map $\mathcal{F}: M_{n} \rightarrow$ $\{0,1\}^{2 n}$ as follows: For all $c \in M_{n}, \mathcal{F}(c)=b=\left(b_{1}, \ldots, b_{2 n}\right)$ where $b_{i}$ is defined as follows: If there is a $k$ such that $c_{1}+\ldots+c_{k}=i$, then $b_{i}=1$ and $b_{i-n+1}=0$. Otherwise, $b_{i}=0$ and $b_{i-n+1}=1$.

Lemma 4.8. $\mathcal{F}$ is one-to-one.

Proof. Assume $\mathcal{F}(x)=\mathcal{F}(y)=w$, and let $j$ be the smallest index where $w_{j}=1$. We have $x_{1}=y_{1}=j$. The next nonzero index will determine that $x_{2}=y_{2}$ and likewise, we can verify that all entries of $x$ and $y$ are equal.

Definition 4.8. Let $\mathcal{B}_{n} \subset\{0,1\}^{2 n}$ be the set of all $b \in\{0,1\}^{2 n}$ such that for all $1 \leqslant i \leqslant n$, $b_{i}+b_{n+i}=1$. We define the action of rotation $\mathcal{R}: \mathcal{B} \rightarrow \mathcal{B}$ on this set as follows: Partition $b$ into maximal blocks of $1^{k} 0$, remove the leftmost block, and put it on the right, ignore partitioning divisions.

Example 4.1. Let $b=(110010001101)$. The partitioning of $b$ will be (110.0.10.0.0.110.1). After swapping the position of last block from (110.0.10.0.0.110.1) to (0.10.0.0.110.1.110), we get $\mathcal{R}(b)=(0.10 \cdot 0.0 .110 .1 .110)$. Note that the block partitioning often changes after each application of rotation.

Lemma 4.9. For any $c \in M_{n}$, we have $\mathcal{F}(C(c))=\mathcal{R}(\mathcal{F}(c))$.

Proof. Consider an arbitrary $c=\left(c_{1}, \ldots, c_{n}\right) \in M_{n}$. Let's say we have $c_{1}=\cdots=c_{k-1}=0$, and $c_{k}$ is the leftmost nonzero element in $c$. Consider the set $\mathcal{A}=\left\{a_{1}=c_{k}, a_{2}=\right.$ $\left.c_{k}+c_{k+1}, \ldots, a_{n-k}=\sum_{i=k}^{n} c_{i}\right\}$. Let $C(c)=c^{\prime}$ and $b=\left(b_{1}, \ldots, b_{n}\right)$, the binary word representing $\mathcal{A}$. In other words for all $a, a \in \mathcal{A}$ if and only if $b_{a}=1$. 
Similarly, let $\mathcal{A}^{\prime}=\left\{c_{k^{\prime}}, c_{k^{\prime}}+c_{k^{\prime}+1}, \ldots, \sum_{i=k^{\prime}}^{n} c_{i}^{\prime}\right\}, k^{\prime}$ being the leftmost nonzero element in $c^{\prime}$ and $b^{\prime}$ the binary presentation of $\mathcal{A}^{\prime}$.

According to definition of crawl we know that if we have $c_{k}=\cdots=c_{l-1}=1$, we will have $c_{k-1}^{\prime}, \ldots, c_{l-2}^{\prime}=0$ and $c_{l-1}^{\prime}=c_{l}-1$, where $l$ is the leftmost element greater than 1 . This means that if have $a_{1}=1, a_{2}=2, a_{3}=3, \ldots a_{l-1}=l$, they should be removed from $\mathcal{A}$ to make $\mathcal{A}^{\prime}$. In other words, any set of consecutive elements starting from a 1 will be removed in $\mathcal{A}^{\prime}$. Moreover, $c_{l}$ will be decremented which means $a_{1}$ and the rest of the elements in $\mathcal{A}$ will be decreased by $l$ except the last one which should always be an $n$. Now, let's see how $b$ will change accordingly. We remove consecutive elements starting with a 1 from $\mathcal{A}$ which means we remove the preceding 1 s from $b$ until we hit a zero. All the other elements will be decreased by $l$ which means they should be shifted left by $l$ positions. This is equivalent to removing the first block from $b$. Now, we need to add $b_{n-l+1}^{\prime}, \ldots, b_{n-1}^{\prime}=0$. And $b_{n}^{\prime}=1$ because $c_{n}^{\prime}$ should be increased by $l$ to make the length of the snake equal to $n$. This whole process is removing the leftmost block and adding its negation to the right, which is equivalent to a rotation of a block in $\mathcal{F}(c)$.

Lemma 4.10. For all $x \in \mathcal{B}_{n}, \mathcal{R}^{n}(x)=x$.

Proof. Consider any arbitrary $x$. Any block in $x$ has a single zero. Moreover, the number of zeros in $x$ is $n$. Therefore, after $n$ rotations $x$ will get back to its initial state.

Proof of Lemma 4.7. From Lemmas 4.10 and 4.9 and the fact that $\mathcal{F}$ is a one-to-one function we conclude that for all $c \in \mathcal{M}_{n}, C^{n}(c)=n$.

\section{Acknowledgements}

This work benefited from helpful discussions with James Propp and Tom Roby. In fact Theorem 3.1, the correspondence between winching and promotion/rowmotion and also the homomesic functions discussed in Section 4 were observed and brought to author's attention by James Propp. I also would like to thank Peter Winkler for his very insightful assistance, and an anonymous reviewer for detailed and helpful comments.

\section{References}

[1] D. Armstrong, C. Stump and H. Thomas, A Uniform Bijection Between Nonnesting and Noncrossing Partitions (August 2013), Transactions of American Mathematical Society, Vol. 365, No. 8, Pages 4121-4151.

[2] J. Bloom, O. Pechenik and D. Saracino, Proofs and Generalizations of a Homomesy Conjecture of Propp and Roby (2016), Discrete Mathematics, Vol. 339, Issue 1, Pages 194-206.

[3] A. Brouwer and A. Schrijver, On the Period of an Operator Defined on Antichains (1974), Stichting Mathematisch Centrum, Zuivere Wiskunde (ZW 24/74): 1-13. 
[4] P. Cameron and D. Fon-Der-Flaass, Orbits of Antichains Revisited (1995), European Journal of Combinatorics, Vol. 16, Issue 6, Pages 545-554.

[5] M. Chan, S. Haddadan, S. Hopkins, and L. Moci, The Expected Jaggedness of Order Ideals, (2017), SIGMA Forum of Mathematics, Vol. 5, Issue 9.

[6] K. Dilks, J. Striker, C. Vorland, Rowmotion and Increasing Labeling Promotion (2019), Journal of Combinatorial Theory, Series A, Vol. 164, Pages 72-108.

[7] D. Einstein, M. Farber, E. Gunawan, M. Joseph, M. Macauley, J. Propp, and S. Rubinstein-Salzedo, Noncrossing Partitions, Toggles, and Homomesies. (2016), DMTCS Proceedings of Discrete Mathematics and Theoretical Computer Science, Pages 419-430.

[8] D. Einstein and J. Propp, Piecewise-linear and Birational Toggling, (2014), Discrete Mathematics \& Theoretical Computer Science (2014), Pages 513-524.

[9] S. Hopkins and I. Zhang, A Note on Statistical Averages for Oscillating Tableaux, (2015), The Electronic Journal of Combinatorics, Vol. 22, Issue 2, \#P2.48.

[10] D. Panyushev, On Orbits of Antichains with Positive Roots (2009), European Journal of Combinatorics, Vol. 30, Issue 2, Pages 586-594.

[11] G. Pólya, Kombinatorische Anzahlbestimmungen fur Gruppen, Graphen und chemische Verbindungen (1937), Acta Mathematica, Vol. 68 (1), Pages 145-254.

[12] J. Bernstein, J. Striker, C. Vorland, P-strict promotion and piecewise linear rowmotion, with applications to tableaux of many flavors, arXiv:2012.12219.

[13] M. Joseph and T. Roby, Birational and non-commutative lifts of anti-chain toggling and row-motion, Algebraic Combinatorics, Vol. 3(4) (2020), Pages. 955-984. arXiv:1909.09658.

[14] J. Propp and T. Roby, Homomesy in Products of Two Chains (2015), DMTCS Proceedings, The Electronic Journal of Combinatorics, Vol. 22, Issue 3, \#P3.4.

[15] D. B. Rush and X. Shi, On Orbits of Order Ideals of Minuscule Posets (2013), Journal of Algebraic Combinatorics: An International Journal, Vol. 37, Issue 3, Pages 545569.

[16] T. Roby, Dynamical Algebraic Combinatorics and the Homomesy Phenomenon (First online: 13 April 2016); Chapter of Recent Trends in Combinatorics; Part of the IMA Volumes in Mathematics and its Applications book series (IMA, Vol. 159).

[17] J. Striker and N. Williams, Promotion and Rowmotion (2012), European Journal of Combinatorics, Vol. 33, Issue 8, Pages 1919-1942.

[18] D. Grinberg, T. Roby, Iterative Properties of Birational Rowmotion I: Generalities and Skeletal Posets (2016), The Electronic Journal of Combinatorics, Vol. 23, Issue 1, Pages 1-33. 